\title{
MEANDERING OF TRAJECTORIES OF POLYNOMIAL VECTOR FIELDS IN THE AFFINE $n$-SPACE
}

\author{
D. Novikov And S. YAKOVEnKO
}

\begin{abstract}
We give an explicit upper bound for the number of isolated intersections between an integral curve of a polynomial vector field in $\mathbb{R}^{n}$ and an affine hyperplane.

The problem turns out to be closely related to finding an explicit upper bound for the length of ascending chains of polynomial ideals spanned by consecutive derivatives.

This exposition constitutes an extended abstract of a forthcoming paper: only the basic steps are outlined here, with all technical details being either completely omitted or at best indicated.
\end{abstract}

\section{Contents}

1. Geometry: Trajectories of polynomial vector fields and their meandering

224

1.1. Formulation of the main result

1.2. Remarks

1.3. Related problems

1.4. Ramifications

2. Analysis: Construction of a quasilinear equation

2.1. Linear $n$th order equation

2.2. The nonlinear multidimensional case: the construction

2.3. Integrality via universality

3. Algebra: Chains of ideals and chains of varieties

3.1. Basic definitions

3.2. Strict monotonicity and convexity

3.3. General finiteness result

3.4. Decreasing chains of algebraic varieties

3.5. Dimension-preserving iterations

3.6. Chains of ideals spanned by consecutive derivatives 


\section{Geometry: Trajectories of polynomial vector fields and their meandering}

An algebraic curve defined by simple formulas in $\mathbb{R}^{n}$ cannot be strongly meandering in the following sense: each (affine) hyperplane in $\mathbb{R}^{n}$ either entirely contains this curve, or intersects it by no more than a finite number of isolated points, and this number can be easily bounded in terms of the algebraic complexity of the curve (the degree of polynomial equations determining it). For example, if the curve is explicitly parameterized as $t \mapsto\left(x_{1}(t), \ldots, x_{n}(t)\right)$, where $x_{j}(t)$ are polynomials of degree $\leqslant d$ in one variable, then the number of isolated intersections with a hyperplane is at most $d$. More generally, if a nonsingular curve is defined as a common zero locus for any finite number of polynomials in $n$ variables of degree $\leqslant d$, then the number of isolated intersections cannot be greater than $d^{n-1}$ (a variation on the theme of Bézout theorem).

It would be natural to expect that an integral curve of a polynomial vector field of degree $\leqslant d$, though almost never algebraic, would exhibit a similar property: the number of isolated intersections with any hyperplane can be explicitly majorized in terms depending only on the differential equations determining the curve. Note that if a solution would be explicitly known, then one might try to use a result from [15] claiming that the number of isolated intersections with any affine hyperplane is no greater than a weighted sum of integral Frenet curvatures. But even for the rare case of integrable equations the answer in terms of integral curvatures may well happen to be not uniformly bounded over all integral curves.

Easy examples show that it is insufficient to know only the degree of the polynomials defining the vector field; the magnitude of the coefficients affects the possible number of intersections already in the linear case. The "size" of the curve also must be taken into account. Besides, there seems to be no more relevant parameters, and the question arises: is it possible to place an explicit upper bound on the number of intersections between an integral curve and a hyperplane, in terms of the dimension of the space, the degree of the vector field, the magnitude of its coefficients and the size of the curve?

An affirmative answer to this question constitutes the main result of this paper.

1.1. Formulation of the main result. Consider a system of $n$ polynomial nonautonomous ordinary differential equations $\dot{x}=v(t, x)$ or, more explicitly, 


$$
\begin{gathered}
\dot{x}_{i}=v_{i}\left(t, x_{1}, \ldots, x_{n}\right), \quad v_{i} \in \mathbb{R}\left[t, x_{1}, \ldots, x_{n}\right], \quad i=1, \ldots, n \\
t \in \mathbb{R}, \quad x=\left(x_{1}, \ldots, x_{n}\right) \in \mathbb{R}^{n}, \quad \operatorname{deg} v:=\max _{i=1, \ldots, n} \operatorname{deg} v_{i} \leqslant d
\end{gathered}
$$

with the polynomial right hand sides $v_{i}$ of some known degree $d$.

Recall that the height $\mathfrak{h}(p)$ of a multivariate polynomial $p$ is the maximal absolute value of its coefficients. We assume that the height of the right hand side of the system (1.1) is known and bounded by a certain constant $r$ :

$$
\begin{gathered}
v_{i}=v_{i}(t, x)=\sum_{j+|\alpha| \leqslant d} v_{i j \alpha} t^{j} x^{\alpha}, \quad \alpha=\left(\alpha_{1}, \ldots, \alpha_{n}\right) \in \mathbb{Z}_{+}^{n}, \\
\mathfrak{h}(v)=\max _{i} \mathfrak{h}\left(v_{i}\right)=\max _{i} \max _{j, \alpha}\left|v_{i j \alpha}\right| \leqslant r .
\end{gathered}
$$

Let $\Gamma$ be a solution to this system, a parameterized curve, whose geometric dimensions are also known: we assume that in the space-time the graph of $\Gamma$ is contained in the ball of some radius $r^{\prime}$ centered at the origin (the minimal such $r^{\prime}$ will be called the height of the curve and denoted by $\mathfrak{h}(\Gamma)$ for aesthetical reasons),

$$
\begin{gathered}
\Gamma: I \rightarrow \mathbb{R}^{n}, \quad I \ni t \mapsto x(t) \in \mathbb{R}^{n}, \\
I \subseteq\left[-r^{\prime}, r^{\prime}\right], \quad\|x(t)\|:=\max _{j=1, \ldots, n}\left|x_{j}(t)\right| \leqslant r^{\prime} \quad \forall t \in I .
\end{gathered}
$$

Our principal goal is to place an explicit upper bound on the number of isolated intersections between $\Gamma$ and an arbitrary affine hyperplane in $\mathbb{R}^{n}$, knowing only the degree and the height of the determining equation (1.1) and the height of the trajectory $\Gamma$. Obviously, it would be sufficient to estimate in the above terms the number of isolated intersections with the hyperplane $\left\{x_{1}=0\right\}$. Moreover, in order to reduce the number of initial data, we assume (and it turns out to be rather natural) that $r=r^{\prime}$. To abbreviate the formulations, we introduce the following notation.

Definition 1. Let $n, d$ be two natural numbers and $r>0$ a real number. The meandering index $\Omega(r, n, d)$ is the supremum of the possible number of isolated intersections with hyperplanes, taken over all polynomial equations of dimension $n$, degree $d$ and height $r$ and over all their integral curves of (the same) height $r$.

One may show, using some general results on real analytic sets [3], that $\Omega(r, n, d)$ is always finite, but the methods used in $[\mathbf{3}]$ give absolutely no 
information on the nature of $\Omega$ as a function of its arguments. Our main result claims computability of $\Omega$ in a certain very strong sense and places an explicit upper bound on it.

Definition 2. A primitive recursive function is a function from $\mathbb{Z}_{+}^{m}$ to $\mathbb{Z}_{+}$that can be obtained from the basic functions (identity and the constant 1) by a finite number of compositions (substitutions), juxtaposition (concatenation) and recursion in one variable. The latter rule allows to construct a function using the recursive construction

$$
\begin{gathered}
f(1, \nu)=g(\nu), \quad \nu=\left(\nu_{1}, \ldots, \nu_{a}\right) \in \mathbb{Z}_{+}^{m}, \\
f(k+1, \nu)=G(k, f(k, \nu), \nu), \quad k=1,2, \ldots,
\end{gathered}
$$

provided that the functions $g(\cdot)$ and $G(\cdot, \cdot, \cdot)$ are already constructed.

Despite the seemingly all-embracing appearance of the class of primitive recursive functions, this class does not include all functions that are computable in the algorithmic sense of this word. As a counterexample, one could mention the Ackermann generalized exponential described in section 3.3. This function grows more rapidly than any primitive recursive function, and in a strange way appears in connection with the problem of bounding $\Omega$, as explained in sections 3.3 and 3.4.

Now the principal result of this paper can be formulated.

\section{Theorem 1 (main).}

$$
\Omega(r, n, d) \leqslant(2+r)^{B(n, d)},
$$

where $B$ is a finite number depending only on $n$ and $d$.

Moreover, $B(n, d)$ as a function of two natural arguments is explicitly computable, admits a primitive recursive majorant and for large $n, d$ grows not more rapidly than the tower of four exponents, $\exp ^{[4]}(n+d) \equiv$ exp exp exp $\exp (n+d)$.

1.2. Remarks. This formulation deserves, to our opinion, several comments. The finiteness of $\Omega(r, n, d)$ is, as already mentioned, a corollary to a very basic property of real analytic sets.

The analytic section 2 provides information sufficient to conclude that for any particular choice of $n$ and $d$, the meandering index $\Omega(r, n, d)$ as a function of $r$ grows at most polynomially. In other words, the value $B(n, d)$ is proved to exist and be always finite. 
In the beginning of section 3 we explain why $B(n, d)$ can be explicitly majorized using some finite deterministic algorithms, and therefore admits a recursive, but not (yet) necessarily primitive recursive majorant. The problem is in estimating the maximal length of certain ascending chains of polynomial ideals, the subject that received substantial attention since the paper by G. Hermann [8] and later works by A. Seidenberg [18], [19].

Five years ago one might hope that upgrading recursiveness to the primitive recursiveness is a matter of technical skill. However, the recent results by G. Moreno Socías [14] show that the best result one can get without additional information is the upper bound in the form of the Ackermann generalized exponential, see below in section 3.3. The growth of this function is faster than any closed expression (elementary function, e.g. the tower of one million exponents) or even any primitive recursive function.

However, one can obtain much better estimate for the length, using the algebraic fact that the ideals in the chain are obtained by adding consecutive derivatives, resulting in a certain "convexity" of the "monotonous" chain of ideals. In section 3 we discuss without going into technical details, how this "convexity" makes ascending chains infinitely shorter, resulting in the tower of only three exponents. This implies that the bound for the original problem on the number of intersections can be achieved as the tower of 4 exponents.

Concluding this informal discussion, we would like to stress that the function $B(n, d)$ can be written in an explicit closed form. Moreover, the asymptotic upper bound given in Theorem 1 is certainly excessive (the price for its relatively compact form). Yet we were unable to reduce it to the tower of two exponents (see section 1.3 below), not saying about polynomial expressions.

1.3. Related problems. A local counterpart of the problem on counting isolated intersections is the problem on estimating the maximal multiplicity of contact, known as Risler problem [17]. An explicit answer in the Risler problem was relatively recently obtained first by A. Gabrielov, J.-M. Lion and R. Moussu for planar $(n=2)$ curves [4], and then by Gabrielov in [2] for the general case: the multiplicity of isolated contact between an integral curve of a polynomial system (1.1) and a hyperplane does not exceed $(d+1)^{2^{n}}$, which makes a tower of two exponents. Note that the bound does not depend on the height(s).

Another problem that leads to similar considerations, appears when one replaces the differential equation (1.1) by a polynomial discrete time dynamical system of the form 


$$
\mathbb{R}^{n} \ni x(t) \mapsto x(t+1)=P(t, x(t)) \in \mathbb{R}^{n}, \quad t=0,1,2, \ldots,
$$

where $P: \mathbb{R} \times \mathbb{R}^{n} \rightarrow \mathbb{R}^{n}$ is a polynomial map of some known degree $d$. For such systems the order of contact between an (infinite) orbit $\{x(0), x(1), x(2), \ldots\}$ and an algebraic hypersurface (say, a hyperplane $\left.X_{0} \subseteq \mathbb{R}^{n}\right)$, is the length of the initial segment of the sequence $\{x(t)\}_{t=0}^{\ell}$ that belongs to $X_{0}$, provided that the infinite orbit leaves $X_{0}$ at a certain moment (otherwise the "contact" between the orbit and the hypersurface is "nonisolated"). The problem that will be referred to as the discrete Risler problem, is to estimate the maximal possible order of an isolated contact, knowning only the dimension $n$ and the degree $d$.

Besides the natural analogy, the discrete Risler problem naturally appears when one tries to solve the (original) Risler problem by expanding solutions in Taylor series, at least in the linear case. On the other hand, the method of proving Theorem 1 is more easy to explain in slightly different settings provided by the analysis of the discrete Risler problem.

1.4. Ramifications. Besides the principal result of Theorem 1, we solve also the discrete Risler problem for sufficiently generic polynomial maps that are dimension-preserving in the sense described below in section 3.5 .

The solution of the discrete Risler problem, after additional considerations similar to that of section 2 and using some very recent results by M. Briskin and Y. Yomdin [1], [20], would allow to estimate the number of isolated intersections with hyperplanes for analytic curves, whose vector Taylor coefficients can be obtained by polynomial recurrent procedures (as in the case of solutions of linear polynomial systems of first order differential equations).

We would also remark that being originally totally real, the question about the number of intersections makes sense also for holomorphic integral curves of polynomial differential equations. Our proof works without any change in the complex settings as well, and the final bound remains the same. The only difference is that the reference to Lemma 1 below should be replaced by that to its complex analog from [16].

Acknowledgements. This work would never be done without help and support of our friends and colleagues who patiently explained us some basics and fine points of commutative algebra. We are grateful to Alexander Braverman, Maria Gorelik, Vladimir Hinich, Anna Melnikov, André Reznikov, Victor Vinnikov, Amnon Yekutieli.

Yosef Yomdin, Vladimir Golubyatnikov, Marie-Françoise Coste-Roy and Andrei Gabriélov were among the first who discussed this problem 
with us and by showing constant and strong interest provided us with additional stimuli.

However, our special and cordial gratitude goes primarily to Joos Heintz: he introduced us into the beautiful realm of effective commutative algebra and taught us many fundamental results. In particular, he supplied us with the explicit estimate of the complexity of primary decomposition that we otherwise would be unable to produce.

\section{Analysis: Construction of a quasilinear equation}

Let $t \mapsto\left(x_{1}(t), \ldots, x_{n}(t)\right)$ be the parametric representation of the curve, $x_{0}(t) \equiv 1$ and $\lambda_{0}, \lambda_{1}, \ldots, \lambda_{n}$ a tuple of real constants. To majorize the meandering, one has to estimate the number of isolated zeros of an arbitrary linear combination $\lambda_{0}(t) x_{0}+\lambda_{1} x_{1}(t)+\cdots+\lambda_{n} x_{n}(t)$. Typically such linear families of functions appear as solutions to higher order linear differential equations (with variable coefficients). Thus we start with our principal example that will also serve as a principal tool in further investigations.

2.1. Linear $n$th order equation. Suppose that a real analytic function $f: I \rightarrow \mathbb{R}$ is known to satisfy on this interval some linear ordinary differential equation of order $\nu$ with real analytic coefficients,

$$
y^{(\nu)}+a_{\nu-1}(t) y^{(\nu-1)}+\cdots+a_{1}(t) y^{\prime}+a_{0}(t) y=0,
$$

and the coefficients $a_{j}(t)$ extend analytically into some open neighborhood $U$ of the real interval $I \Subset \mathbb{R} \subseteq \mathbb{C}$ in the complex plane. Then the upper bound for the absolute value of the coefficients in $U$ is naturally referred to as the height of the equation (2.1). It turns out that any solution to an equation of bounded height may have only a bounded number of isolated zeros. The precise formulation follows.

Lemma 1 (see [9, Theorem 1]). If $\left|a_{j}(t)\right| \leqslant r$ in $U \ni I$, then any real solution of the equation (2.1) may have no more than $\gamma \cdot(\nu+r)$ isolated zeros on $I$, where $\gamma=\gamma(I, U)<+\infty$ is an explicit "geometric" constant determined by the relative position of I inside $U$.

Note that one should not assume that the coefficients $a_{j}$ are polynomial or rational, though in many cases they turned to be: only their height $r$ is essential. The asymptotic behavior of the geometric constant $\gamma(I, U)$ is studied in [9, section 1.2].

The idea behind the proof of Lemma 1 is rather simple: the number of isolated zeros of an analytic function is related via the Jensen inequality 
to the growth of this function in the gap between $I$ and $U$. On the other hand, solutions of linear equations cannot grow too rapidly if the coefficients of the equations are explicitly bounded.

2.2. The nonlinear multidimensional case: the construction. The problem of estimating $\Omega(r, n, d)$ is equivalent to estimating the number of zeros of $x_{1}$-coordinate along an arbitrary solution of the system (1.1). In this section we construct explicitly a linear equation of the form (2.1) satisfied by the function $y(t)=x_{1}(t)$.

Let $\mathfrak{R}=\mathbb{C}[t, x]=\mathbb{C}\left[t, x_{1}, \ldots, x_{n}\right]$ be the ring of polynomials in $t$ and $x$; denote by $D: \mathfrak{R} \rightarrow \mathfrak{R}$ the derivation along the system (1.1): $D=$ $\partial_{t}+\sum_{i=1}^{n} v_{i}(t, x) \partial_{i}, \partial_{i}=\partial / \partial x_{i}, i=1, \ldots, n$. Consider the sequence of polynomials $\left\{p_{k}\right\}_{k=0}^{\infty} \subseteq \mathfrak{R}$ defined by the initial condition $p_{0}(x)=x_{1} \in \mathfrak{R}$ and the recursive rule

$$
\begin{aligned}
& p_{0}=x_{1}, \\
& p_{k}=D p_{k-1}, \quad k=1,2,3, \ldots .
\end{aligned}
$$

The ring $\mathfrak{R}$ is Noetherian, therefore the ascending chain of ideals

$$
\begin{aligned}
(0) \subseteq\left(p_{0}\right) \subseteq & \left(p_{0}, p_{1}\right) \subseteq\left(p_{0}, p_{1}, p_{2}\right) \subseteq \ldots \\
& \cdots \subseteq\left(p_{0}, p_{1}, \ldots, p_{k}\right) \subseteq\left(p_{0}, p_{1}, \ldots, p_{k}, p_{k+1}\right) \subseteq \ldots
\end{aligned}
$$

must stabilize at a certain moment $\ell$ so that $p_{\ell} \in\left(p_{0}, \ldots, p_{\ell-1}\right)$ and hence

$$
p_{\ell}=h_{\ell-1} p_{\ell-1}+\cdots+h_{1} p_{1}+h_{0} p_{0}, \quad h_{j} \in \Re .
$$

Consider now any trajectory $\Gamma: t \mapsto x(t)$ of the nonlinear system (1.1). By construction, the polynomial $p_{k}=p_{k}(t, x)$ restricted on this curve, is the $k$ th derivative $\frac{d^{k}}{d t^{k}} x_{1}(t)$, while the restriction of $h_{k}$ becomes an analytic function that we denote by $-a_{j}(t)$. Altogether, the identity (2.4) would mean then that the function $y(t)=x_{1}(t)$ along $\Gamma$ satisfies the linear equation (2.1) of order $\ell$. By Lemma 1 , to estimate the number of zeros it would be sufficient to know the height of the coefficients on some complex neighborhood of the real segment $I$ and the order of the equation $\ell$.

The problem on estimating $\ell$ is a rather difficult problem that will be treated separately in section 3 and in particular in section 3.6. But even knowing $\ell$ is in general not sufficient to estimate the heights of the polynomials $h_{j}$ in (2.4). Indeed, the identity (2.4) means a system of linear equations for the coefficients of the polynomials $h_{j}$. This system 
is known to admit at least one solution, but nobody can guarantee that this system is sufficiently well-posed so that in an attempt to solve it one never divides by numbers too close to zero.

One case when such guarantee can be given is when all polynomials $p_{j}$ are with integral coefficients. Then all nonzero denominators will be at least equal to 1 in the absolute value, and the division will be well-posed. These arguments are in more details exposed below.

2.3. Integrality via universality. The key idea behind the second step is to ensure that all polynomials $p_{k}$ have integral coefficients. This would allow to find a representation (2.4) of some bounded height. The required integrality is achieved by adjoining formally all coefficients of the polynomials $v_{j}$ to the list of independent variables.

More precisely, we consider the full collection of coefficients $\left\{v_{i j \alpha}\right\}$ of all polynomials $v_{i}(t, x)$ as described in (1.2) as new independent variables, and denote by $\Lambda=\left\{\lambda_{1}, \ldots, \lambda_{n^{\prime}}\right\}=\left\{t, x_{1}, \ldots, x_{n}, \ldots, v_{i j \alpha}, \ldots\right\}$, the combined list of all variables of length $n^{\prime}=1+n+n \cdot\left(\begin{array}{c}n+d \\ n\end{array}\right)$. Denote by $\mathfrak{R}^{*}$ the extended polynomial ring, $\mathfrak{R}^{*}=\mathbb{C}[\Lambda]=\mathbb{C}\left[t, x_{j}, v_{i j \alpha}\right]$.

Then the derivation $D$ extends (by the same formula) to the derivation $D^{*}: \mathfrak{R}^{*} \rightarrow \mathfrak{R}^{*}$ and the sequence of polynomials arises as in (2.2). Actually, the polynomials will be the same, only their dependence on the coefficients of the vector field $v$ will now be explicit.

In other words, we consider (1.1)-(1.2) as a vector field in the space with coordinates $\left(t, x_{j}, v_{i j \alpha}\right)$, on understanding that the remaining coordinates are governed by the trivial equations

$$
\dot{t}=1, \quad \dot{v}_{i j \alpha}=0 \quad 1 \leqslant i \leqslant n, 0 \leqslant j+|\alpha| \leqslant d .
$$

Notice that with respect to the new set of independent variables:

(1) the extended derivation $D^{*}$ is integral, i.e. all right hand sides $v_{i}^{*}$ of the corresponding system of equations are integral polynomials from the subring $\mathbb{Z}[\Lambda]=\mathbb{Z}\left[t, x_{j}, v_{i j \alpha}\right] \subset \mathfrak{R}^{*}$ (actually, the coefficients are only 0 's or 1 's),

(2) the degrees of these polynomials do not exceed $d+1$ and their height is 1 ,

(3) the polynomials $p_{k}$ are also integral, with their degrees growing linearly in $k$ and heights roughly exponential in $k$.

The chain (2.3) understood now as a chain of ideals in $\mathfrak{R}^{*}$, is determined by the numbers $n$ and $d$ only, therefore one has one and the same bound $\ell=\ell(n, d)$ for the length of this chain, uniform over all problems of the given dimension and degree. This already implies the existential upper 
bound of the form $\Omega(r, n, d) \leqslant(2+r)^{B(n, d)}$ with some finite power $B$ depending only on $n$ and $d$, as the identities (2.4) also become universal.

Assuming the number $\ell(n, d)$ being known, we show how the integrality can be used to estimate the magnitude of the coefficients of the quasilinear equation (2.1) resulting from (2.4).

Lemma 2. The representation (2.4) can be chosen in such a way that the degrees $\operatorname{deg} h_{j}$ and the heights $\mathfrak{h}\left(h_{j}\right)$ admit primitive recursive majorants depending on $n, d$ and $\ell$.

If one does not try to get the best known bound, then this result is a rather simple observation modulo a nontrivial theorem by G. Hermann [8]. This theorem claims that if a polynomial $p_{\ell}$ belongs to the ideal spanned by $p_{0}, \ldots, p_{\ell-1} \in \mathbb{C}[\Lambda]$, then one can find a representation of the form (2.4) such that

$$
\begin{aligned}
& \operatorname{deg} h_{j} p_{j} \leqslant \operatorname{deg} p_{\ell}+2\left(2 \max \operatorname{deg} p_{j}\right)^{2^{n^{\prime}-1}} \\
& \qquad \text { for all } j=0,1,2, \ldots, \ell-1,
\end{aligned}
$$

where $n^{\prime}=\operatorname{dim} \Lambda$ is the effective number of variables (including the artificial ones). Since the degrees of all $p_{j}$ are explicitly known, the inequalities (2.5) allow to place an upper bound on $\operatorname{deg} h_{j}$ which was shown in $[\mathbf{1 3}]$ to be essentially sharp.

To estimate the heights of the coefficients, we use the method of indefinite coefficients, writing $\mathfrak{R}^{*} \ni h_{k}=\sum_{|\alpha| \leqslant N} h_{k \alpha} \lambda^{\alpha}$, where $h_{k \alpha}$ are unknown coefficients. Their number is explicitly bounded as the degrees $\operatorname{deg} h_{j}$ are bounded, and the identity (2.4) becomes a linear nonhomogeneous system of (algebraic) equations, with the coefficients of this system and the free terms being explicitly bounded. In general this would not be sufficient to estimate the magnitude of the solutions, since the matrix of coefficients can be very badly invertible. However, if all coefficients were integral, then all nonzero minors of the matrix are $\geqslant 1$ in the absolute value, which fact yields an upper bound on the height of solutions, and this bound obviously admits a primitive recursive majorant. There are substantially better estimates on the height of the coefficients, but the upper bound on $\ell$, discussed below, is so large that the difference between more and less accurate estimates will finally disappear on the level of the principal asymptotics.

Next, knowing the height of $h_{k}$, one can explicitly estimate the height of the resulting equation (2.1) on the ball of radius $2 r$ and find an explicit lower bound for the positive $\varepsilon$, the radius of the neighborhood $U$ onto which the coefficients can be analytically extended. This proves the following corollary. 
Corollary 1. The coefficients of the equation (2.1) constructed via the identity (2.4), extend to some complex $\varepsilon$-neighborhood of the real segment $I$ with guaranteed $\varepsilon \geqslant(2+r)^{-C(n, d, \ell)}$. Moreover, these extended coefficients admit a polynomial in $r$ upper bound of the form $\left|a_{j}\right| \leqslant(2+$ $r)^{B^{\prime}(n, d, \ell)}$ in this $\varepsilon$-neighborhood. The exponents $C, B^{\prime}$ admit primitive recursive majorants.

Thus the problem of estimating the meandering number $\Omega(r, n, d)$ is reduced to studying ascending chains of ideals. As soon as the length is shown to be a primitive recursive function of $n$ and $d$, Theorem 1 would follow from the above corollary.

\section{Algebra: Chains of ideals and chains of varieties}

The fact that the chain (2.3) of polynomial ideals stabilizes, reflects the most fundamental property of polynomial rings. However, effective estimation of the length of such chain is a rather difficult problem. In what follows we denote by $I_{k} \subseteq \Re$ the ideal spanned by $p_{0}, \ldots, p_{k}$, the sequence $p_{k}$ being defined as in (2.2).

3.1. Basic definitions. We list several standard definitions. For two ideals $I, J \subseteq \mathfrak{R}$ their colon ratio is the ideal defined as $I: J=$ $\{p \in \mathfrak{R}: p J \subseteq I\}$. The dimension of an ideal is the (complex analytic) dimension of the zero locus $V(I)=\left\{x \in \mathbb{C}^{n}: p(x)=0 \forall p \in I\right\}$. This analytic dimension coincides with the Krull dimension defined in algebraic terms [21].

If a point $a \in \mathbb{C}^{n}$ is a complex isolated point of $V(I)$, then the multiplicity of $I$ at $a$ is the (finite) number $\mu_{a}(I)=\operatorname{dim}_{\mathbb{C}} \mathfrak{R}_{\mathfrak{m}_{a}} / I \cdot \mathfrak{R}_{\mathfrak{m}_{a}}$, where $\mathfrak{m}_{a} \subseteq \mathfrak{R}$ is the maximal ideal of the point, $\mathfrak{R}_{\mathfrak{m}_{a}}$ the localization of $\mathfrak{R}$ by $\mathfrak{m}_{a}$ and the ideal $I \cdot \mathfrak{R}_{\mathfrak{m}_{a}}$ is the span of the original ideal $I$ in the local ring $\mathfrak{R}_{\mathfrak{m}_{a}}$.

If $I \subseteq \mathfrak{R}$ is a polynomial ideal of dimension $s$ and $a \in V(I)$ a regular (smooth) point on the zero locus, then a generic affine subspace $\Pi \subseteq \mathbb{C}^{n}$ of codimension $s$ passing through $a$ is transversal to $V(I)$, therefore $a$ will be isolated in $V(I) \cap \Pi$. Hence the multiplicity of the ideal $I+L$ at $a$ is well defined in the above sense (here $L$ is the ideal of the subspace). One can show that this multiplicity (that will be also denoted by $\mu_{a}(I)$ ) is independent of $\Pi$ as soon as the latter remains transversal to $V(I)$.

An ideal $Q \subseteq \mathfrak{R}$ is primary, if $p q \in Q$ and $p \notin Q$ implies that $q^{n} \in$ $Q$ for some $n$. The radical $P=\sqrt{Q}$ of a primary ideal is called the associated prime. If $Q$ is primary, then for almost all points $a \in V(Q)$ 
the multiplicity $\mu_{a}(Q)$ is the same; we refer to it then as simply the multiplicity of $Q$.

After all these definitions are recalled, we proceed with the main property of chains generated by consecutive derivatives.

3.2. Strict monotonicity and convexity. Though for our purposes it would be sufficient to find the first step when $I_{\ell-1}=I_{\ell}$, it turns out that after the first coincidence the chain (2.3) will identically stabilize from that moment on. This follows from a more general though very simple observation.

Lemma 3. The colon ratios $I_{k}: I_{k+1}$ constitute an increasing chain:

$$
\left(I_{0}: I_{1}\right) \subseteq\left(I_{1}: I_{2}\right) \subseteq \cdots \subseteq\left(I_{k-1}: I_{k}\right) \subseteq\left(I_{k}: I_{k+1}\right) \subseteq \cdots
$$

Indeed, taking an arbitrary polynomial $q \in I_{k-1}: I_{k}=I_{k-1}:\left(p_{k}\right)$ we can write $q p_{k}=\sum_{j=0}^{k-1} h_{j} p_{j}$. Applying the derivation $D$, we conclude that $q p_{k+1}=q \cdot D p_{k} \in\left(p_{0}, \ldots, p_{k}\right)=I_{k}$, which means that $q \in I_{k}$ : $\left(p_{k+1}\right)=I_{k}: I_{k+1}$.

A monotonous (ascending) chain of ideals $\left\{I_{k}\right\}$ possessing the property (3.1) will be called a convex chain (as the first "differences" are monotonously growing).

Corollary 2. If the chain (2.3) is convex, then:

(1) it is strictly ascending, i.e. if $I_{\ell-1}=I_{\ell}$, then $I_{\ell}=I_{\ell+1}=$ $I_{\ell+2}=\cdots ;$

(2) the dimensions of the colon ideals in (3.1) are nonincreasing.

Indeed, in this case $I_{\ell-1}: I_{\ell}=(1)=\mathfrak{R}$, which implies that all other colon ideals in (3.1) coincide with the ring $\mathfrak{R}$ and hence $I_{k}=I_{k+1}$ for all $k \geqslant \ell$.

3.3. General finiteness result. The degrees of the polynomials spanning the ideal $I_{k}$ are known to grow linearly in $k$, as seen from the rule $(2.2)$ : $\operatorname{deg} p_{k} \leqslant k(d-1)+1 \leqslant k d$ for $k \geqslant 1$. One might try to place an upper bound on the length of the chain (2.3), using only the information on the degrees. This is possible in principle: in [18], [19] an algorithm is given for computing the length $\ell=\ell(n, d)$ of the ascending chain (2.3) with $\operatorname{deg} p_{k}=k d, \operatorname{dim} x=n$, and the answer is therefore a well defined and computable function of the integer data $n$ and $d$.

However, a detailed analysis recently carried out by G. Moreno [14] shows that in general the length of an ascending chain (2.3) as a function 
of $n$ and $d$ can be as large as the Ackermann generalized exponential, a function that is known to grow faster than any primitive recursive function. From the computational point of view this means that the problem of estimating length is untreatable. Recall that the Ackermann generalized exponential $A(z, x, y)$ is determined by the recursive rules

$$
\begin{aligned}
A(0, x, y) & =y+1, \\
A(1, x, 0) & =x, \\
A(2, x, 0) & =0, \\
A(3, x, 0) & =A(4, x, 0)=\cdots=1, \\
A(z+1, x, y+1) & =A(z, x, A(z+1, x, y)) .
\end{aligned}
$$

In particular,

$$
\begin{aligned}
& A(1, x, y)=x+1+\cdots+1=x+y, \\
& A(2, x, y)=x+x+\cdots+x=x y, \\
& A(3, x, y)=x x \cdots x=x^{y}, \\
& A(4, x, y)=\underbrace{x^{x \cdot{ }^{x}}}_{y \text { times }}
\end{aligned}
$$

etc.

In the remaining part of this section we show that the chains satisfying the convexity condition (3.1), must stabilize infinitely sooner than in the general case. The arguments become more transparent if one considers a closely related problem on lengths of decreasing chains of algebraic varieties.

3.4. Decreasing chains of algebraic varieties. A related but more simple question is about the length of a descending chain of algebraic varieties $X_{k}$, the zero loci of the ideals from the chain (2.3): $X_{k}=\left\{x \in \mathbb{C}^{n}: p_{0}(x)=\cdots=p_{k}(x)=0\right\}$ (obviously, $X_{k+1} \subseteq X_{k}$ ).

If the polynomials $p_{k}$ spanning the ideals $I_{k}$ are consecutive derivatives, then in general it is not true that the decreasing chain is strictly decreasing (example: $n=1, p_{0}=x^{\mu}, D=\partial_{x}, I_{k}=\left(x^{\mu-k}\right), X_{k}=\{0\}$ for $k=0,1, \ldots, \mu-1$ but $\left.I_{\mu}=(1), X_{\mu}=\varnothing\right)$.

However, if the sequence $\left\{p_{k}\right\}_{k=0}^{\infty}$ is generated by iterations of a homomorphism, then the chain of varieties must be strictly decreasing. More precisely, assume that $P: \mathbb{C}^{n} \rightarrow \mathbb{C}^{n}$ is a polynomial map, $H=P^{*}: \mathfrak{R} \rightarrow$ $\mathfrak{R}$ the corresponding ring homomorphism, and instead of the rule $(2.2)$ one has

(3.2) $p_{k+1}=p_{k} \circ P$ or, what is the same, $p_{k+1}=H p_{k}, k=0,1,2, \ldots$. 
Then one can easily prove the following geometric analog of the algebraic assertion of Lemma 3 .

\section{Lemma 4.}

$$
P\left(X_{k} \backslash X_{k+1}\right) \subseteq X_{k-1} \backslash X_{k} \quad \forall k=1,2, \ldots
$$

Indeed, the rule (3.2) allows to interpret $X_{k}$ as the set of points $x$ such that the first $k$ points of the $P$-orbit of $x$ belong to the variety $X_{0}$. This observation links the problem of estimating the length of the descending chain with the discrete Risler problem, see section 1.3. Then the assertion of Lemma 4 becomes obvious: the difference $X_{k} \backslash X_{k+1}$ consists of points that leave $X_{0}$ on the step number $k+1$ and not earlier, while application of $P$ means a shift along the orbit. Since the image of a non-empty set is non-empty (recall that $P$ is a polynomial map defined everywhere), we have the following corollary.

Corollary 3. Under assumption (3.2) the chain $\left\{X_{k}\right\}$ is strictly descending: if $X_{\ell-1}=X_{\ell}$, then $X_{\ell}=X_{\ell+1}=X_{\ell+2}=\cdots$.

The rule (3.2) allows to estimate the degrees $\operatorname{deg} p_{k}$ in the same way as the rule (2.2), the only difference is that the degrees grow as a geometric progression in this case.

The primary tool for studying descending chains of algebraic varieties is the irreducible decomposition: any algebraic variety can be uniquely represented as an irredundant union of irreducible algebraic (sub)varieties of various dimensions. The irreducibility means, among other properties, that a proper subset of an irreducible $s$-dimensional variety has a strictly inferior dimension $s^{\prime}<s$.

An algebraic variety in $\mathbb{C}^{n}$ defined by an arbitrary number of polynomial equations of degree $\leqslant d$, may have no more than $d^{n-s}$ irreducible components of dimension $\geqslant s$. This is a relatively simple generalization of the Bézout theorem [7].

The length of a descending chain of algebraic varieties can be bounded from above by monitoring irreducible components: each component of $X_{k}$ may either belong to $X_{k+1}$, or be split into some number of components of smaller dimensions. The number of such newly-born components is estimated by the Bézout theorem and is proportional to the degree $\operatorname{deg} p_{k+1}$. The worst scenario occurs if on each step only one irreducible component of $X_{k}$ is split, and if this component is of minimal possible dimension. Thus we first see as all 0-dimensional components (isolated points) disappear. Then one of 1-dimensional components (curves) is 
split into a number of points. For some number of subsequent steps these new points will disappear one by one, until the next 1-dimensional component would have to be split etc. Note that in this process the degrees of the polynomials $p_{k}$ grow, thus each next 1-dimensional curve will be split into bigger and bigger number of isolated points.

When the entire original supply of 1-dimensional curves will be over, some 2-dimensional irreducible component should be split, giving rise to a (very large, since the number of the step is already rather large) number of 1-dimensional curves. Then, according to our scenario, we must return to the previous policy of eliminating points and curves, until all of them disappear. The number of steps required for that will be incomparably larger than in the first round.

Even without making accurate computations it is clear that this sort of double induction leads to an overwhelmingly rapidly growing estimates, in fact, to some form of the Ackermann generalized exponential. Of course, one might ask why the worst scenario leading to Ackermann, is realizable, but we will refer the reader to the papers by Moreno [14], where the similar arguments are analyzed for ideals. Moreno proves that the Ackermann generalized exponential gives a sharp estimate of the length of an ascending chain, provided that there is no additional restrictions except for those on the degrees of the polynomials $p_{k}$. Moreover, a minor modification of the construction from [14] yields the proof for decreasing chains of varieties.

3.5. Dimension-preserving iterations. The possible additional property that allows to lift the curse of Ackermann in the problem on descending chains of varieties, is preservation of dimension by iterations of the map $P$.

Definition 3. A polynomial map $P: \mathbb{C}^{n} \rightarrow \mathbb{C}^{n}$ is dimension-preserving, if the image of any $k$-dimensional semialgebraic set is again $k$-dimensional for any $k$ between 0 and $n$.

The additional assumption that $P$ is dimension-preserving, together with the observation from Lemma 4, improves the situation radically: the up'n'down wanderings on the dimension scale, that led to the Ackermann-large bound for the length, are no longer possible.

Lemma 5. If $P$ is a dimension-preserving map and $\left\{X_{k}\right\}$ is a strictly descending chain constructed as in (3.2), then this chain can be subdivided into $\leqslant n$ segments of finite length, 


$$
\begin{array}{r}
\underbrace{X_{0} \supset X_{1} \supset \cdots \supset X_{k_{n-1}}}_{\operatorname{dim} X_{k} \backslash X_{k+1}=n-1} \supset \underbrace{X_{k_{n-1}+1} \supset \cdots \supset X_{k_{n-2}}}_{\operatorname{dim} X_{k} \backslash X_{k+1}=n-2} \supset \cdots \\
\cdots \supset \underbrace{X_{k_{s}+1} \supset \cdots \supset X_{k_{s-1}}}_{\operatorname{dim} X_{k} \backslash X_{k+1}=s} \supset \cdots \supset \underbrace{X_{k_{1}+1} \supset \cdots \supset X_{k_{0}}}_{\operatorname{dim} X_{k} \backslash X_{k+1}=0}
\end{array}
$$

such that along sth segment the differences $X_{k} \backslash X_{k+1}$ are exactly $s$ dimensional semialgebraic varieties.

The length of each such segment does not exceed the number of $s$ dimensional irreducible components in the starting set $X_{k_{s}+1}$ of this segment.

This is almost obvious: the dimensions $\operatorname{dim} X_{k} \backslash X_{k+1}$ must be nonincreasing, hence the chain can be partitioned into segments as required. Along each segment one has to monitor only $s$-dimensional irreducible components, and moreover, their number must strictly decrease on each step inside the segment. Indeed, inside the $s$ th segment all components of dimension $>s$ must be preserved, otherwise the difference will be more than $s$-dimensional. On the other hand, if all $s$-dimensional components are preserved on some step, this means that the difference $X_{k} \backslash X_{k+1}$ is at most $(s-1)$-dimensional, and one starts the next segment.

It remains only to observe that the number of irreducible components can be estimated by the Bézout theorem, and hence for the lengths $k_{s}-k_{s-1}$ we have a recurrent inequality,

(3.5) $k_{s-1}-k_{s} \leqslant\left(d^{k_{s}}\right)^{n-1}=\left(d^{n-1}\right)^{k_{s}}, \quad s=n-1, \ldots, 1,0, \quad k_{n}=0$.

As a result, we obtain a simple upper bound that is infinitely better than the Ackermann generalized exponential: the above inequality can be transformed into $k_{s-1} \leqslant\left(1+d^{n-1}\right)^{k_{s}}$, which obviously gives a tower of $n$ exponents after $n$ iterations. In terms of the discrete Risler problem this result looks as follows.

Theorem 2. If $P: \mathbb{C}^{n} \rightarrow \mathbb{C}^{n}$ is a dimension-preserving polynomial map of degree $\leqslant d$ then for any algebraic hypersurface $X_{0}$ of degree $\leqslant d$ and any point $x \in X_{0}$ the $P$-orbit $\left\{P^{k}(x)\right\}_{k=0}^{\infty}$ either leaves $X_{0}$ no later than on the Nth step, where

$$
N \leqslant \underbrace{A^{A^{.} \cdot{ }^{A}}}_{n-1 \text { times }}, \quad A=1+d^{n-1},
$$


or remains on $X_{0}$ forever.

In other words, the answer is given by the Ackermann function of rank 4, and this is related to the exponential growth of degrees of $p_{k}$ : if the degrees were growing linearly with $k$, then the result would be given by the Ackermann function of rank 3, or, more exactly, by a double exponential in $n$ estimate, as in [2]. In this sense all towers of exponents of some universal height (2, 3 or 1000) all occupy an intermediate place between the Ackermann functions of ranks 3 and 4, "closer" to the former one. Thus the difference between the tower of height 4 that occurs in Theorem 1 and that of height 2 typical for the (continuous) Risler problem, is "negligeable".

3.6. Chains of ideals spanned by consecutive derivatives. Now we briefly describe how the constructions of section 3.5 should be modified for working with chains of ideals. As before, the main tool is the irreducible decomposition of polynomial ideals [21]: any polynomial ideal $I \subseteq \Re$ admits an irredundant representation $I=Q_{1} \cap \cdots \cap Q_{k}$, where $Q_{j} \subseteq \Re$ are primary ideals with pairwise different associated prime ideals.

Additional problems arising when replacing varieties by ideals, are of two kinds. First, the primary decomposition is in general non-unique, except for primary components of maximal dimension [21]. Second, one has to take multiplicities into account, since there may occur a strictly ascending chain of primary ideals with the same associated prime, and one has to look for a numeric indicator of its ascent. The problem with defining multiplicities is rather delicate: in particular, we tried to avoid defining and using the multiplicity for embedded primary components, i.e. those whose associated prime contains the associated prime(s) of some other primary components. This difficulty was circumvented by constructing an arbitrary (not canonically defined) primary decomposition and deleting out of it all primary components of larger dimensions.

The following proposition is an algebraic analog of Lemma 5.

Lemma 6. If $D$ is a derivation and $\left\{I_{k}\right\}$ is a strictly ascending chain constructed as in (2.2), then this chain can be subdivided into $\leqslant n$ segments of finite length,

$$
\begin{gathered}
\underbrace{I_{0} \subset I_{1} \subset \cdots \subset I_{k_{n-1}}}_{\operatorname{dim} I_{k}: I_{k+1}=n-1} \subset \underbrace{I_{k_{n-1}+1} \subset \cdots \subset I_{k_{n-2}}}_{\operatorname{dim} I_{k}: I_{k+1}=n-2} \subset \cdots \\
\cdots \subset \underbrace{I_{k_{s}+1} \subset \cdots \subset I_{k_{s-1}}}_{\operatorname{dim} I_{k}: I_{k+1}=s} \subset \cdots \subset \underbrace{I_{k_{1}+1} \subset \cdots \subset I_{k_{0}}}_{\operatorname{dim} I_{k}: I_{k+1}=0}
\end{gathered}
$$


such that along sth segment the colon ratios $I_{k}: I_{k+1}$ are exactly $s$ dimensional.

Consider any primary decomposition of $I_{k_{s}+1}$ and collect the terms in such a way that $I_{k_{s}+1}=Q_{s}^{\prime} \cap Q_{s}^{\prime \prime}$, where $Q_{s}^{\prime}$ is the intersection of primary components of dimension $>s$ and $Q_{s}^{\prime \prime}$ is the intersection of primary components of dimensions $\leqslant s$. Then $s$-dimensional components of $Q_{s}^{\prime \prime}$ are well defined together with their multiplicities by the choice of $Q_{s}^{\prime \prime}$, and the length of the sth segment does not exceed the number of s-dimensional primary components of $Q_{s}^{\prime \prime}$, counted with their multiplicities.

Note that the decomposition $I_{k_{s}+1}=Q_{s}^{\prime} \cap Q_{s}^{\prime \prime}$ is not uniquely defined, however, any such representation can be used to estimate the length of the $s$ th segment.

Moreover, one has no simple formulas as in Bézout theorem, for the number of irreducible components. Therefore we had to use an algorithm for the effective primary decomposition of a polynomial ideal, and analyze its complexity. This subject, covered in a range of recent publications $[\mathbf{5}],[\mathbf{6}],[\mathbf{1 0}],[\mathbf{1 1}]$, is still too technical to be included here. What we need from this highly developed theory is an explicit upper bound for the degrees of generators and the number of irreducible primary components of an ideal given by a system of generators of known degrees. After this the problem of majorizing multiplicities becomes relatively simple, and we can write a system of recurrent inequalities majorizing the length of segments in (3.6).

\section{References}

1. M. Briskin And Y. Yomdin, Algebraic families of analytic functions I, Preprint, Weizmann Institute of Science (1995).

2. A. Gabrielov, Multiplicities of zeros of polynomials on trajectories of polynomial vector fields and bounds on degree of nonholonomy, Math. Res. Lett. 2 (1996), 437-451.

3. A. Gabrielov, Projections of semianalytic sets, Funktsional. Anal. i Prilozhen. 2(4) (1968), 18-30 (Russian), English transl. in Functional Anal. Appl. 2 (1968), 282-291.

4. A. Gabrielov, J.-M. Lion and R. Moussu, Ordre de contact de courbes intégrales du plan, C.R. Acad. Sci. Paris Sér. I Math. 319(3) (1994), 219-221.

5. P. Gianni, B. Trager and G. Zacharias, Gröbner bases and primary decomposition of polynomial ideals. Computational aspects 
of commutative algebra, J. Symbolic Comput. 6(2-3) (1988), 149167.

6. M. Giusti, "Some effectivity problems in polynomial ideal theory," EUROSAM 84 (Cambridge, 1984), Lecture Notes in Comput. Sci. 174, Springer, Berlin-New York, 1984, pp. 159-171.

7. J. Heintz, Definability and fast quantifier elimination in algebraically closed fields, Theoret. Comput. Sci. 24(3) (1983), 239-277.

8. G. Hermann, Die Frage der endlich vielen Schritte in der Theorie der Polynomialideale, Math. Ann. 95 (1926), 736-788.

9. YU. IL'YASHENKO AND S. YAKOVENKO, Counting real zeros of analytic functions satisfying linear ordinary differential equations, $J$. Differential Equations 126(1) (1996), 87-105.

10. T. KRICK AND A. LogAR, An algorithm for the computation of the radical of an ideal in the ring of polynomials, in "Applied algebra, algebraic algorithms and error-correcting codes," (New Orleans, LA, 1991), Lecture Notes in Comput. Sci. 539, Springer, Berlin, 1991, pp. 195-205.

11. D. LAZARD, A note on upper bounds for ideal-theoretic problems, J. Symbolic Comput. 13(3) (1992), 231-233.

12. S. Lojasiewicz, "Introduction to Complex Analytic Geometry," Birkhäuser, Basel-Boston-Berlin, 1991.

13. E. W. MaYr And A. R. Meyer, The complexity of the word problems for commutative semigroups and polynomial ideals, $A d v$. Math. 46 (1982), 305-329.

14. G. Moreno Socías, Length of polynomial ascending chains and primitive recursiveness, Math. Scand. 71(2) (1992), 181-205. Autour de la fonction de Hilbert-Samuel (escaliers d'idéaux polynomiaux), Ph.D. Thesis, Centre de Mathématiques de l'École Polytechnique, 1991.

15. D. Novikov And S. YAKOvenko, Integral Frenet curvatures and oscillation of spatial curves around affine subspaces of a Euclidean space, J. of Dynamical and Control Systems 2(2) (1996), 157-191.

16. D. Novikov And S. Yakovenko, A complex analog of Rolle theorem and polynomial envelopes of irreducible differential equations in the complex domain, J. London Math. Soc. (1996) (to appear).

17. J.-J. RisLER, A bound for the degree of nonholonomy in the plane, Algorithmic complexity of algebraic and geometric models (Creteil, 1994), Theoret. Comput. Sci. 157(1) (1996), 129-136.

18. A. Seidenberg, Constructions in algebra, Trans. Amer. Math. Soc. 197 (1974), 273-313. 
19. A. Seidenberg, Constructive proof of Hilbert's theorem on ascending chains, Trans. Amer. Math. Soc. 174 (1972), 305-312; On the length of a Hilbert ascending chain, Proc. Amer. Math. Soc. 29 (1971), 443-450.

20. Y. Yomdin, Oscillation of analytic curves, Preprint, Weizmann Institute of Science (1995).

21. O. Zariski And P. SAmuel, "Commutative Algebra," vol. 1, Springer-Verlag, N. Y. et al., 1975, corrected reprinting of the 1958 edition..

Keywords. Oscillation, Ascending chains, Polynomial ideals, Zeros of analytic functions.

1991 Mathematics subject classifications: 14Q05, 30C15, 34C20, 13E05, $13 \mathrm{P} 10$.

Department of Theoretical Mathematics

The Weizmann Institute of Science

Rehovot 76100

ISRAEL

e-mail: dmitri@wisdom.weizmann.ac.il

e-mail: yakov@wisdom.weizmann.ac.il

WWW Home Page: http://www.wisdom.weizmann.ac.il/ yakov/index.html

Rebut el 30 de Novembre de 1996 